\title{
Astrometric versus Spectroscopic Radial Velocities ${ }^{1}$
}

\author{
Dainis Dravins, Dag Gullberg, Lennart Lindegren and Søren Madsen \\ Lund Observatory, Box 43, SE-22100 Lund, Sweden
}

\begin{abstract}
The apparent radial velocity of a star, as deduced from wavelength shifts, comprises not merely its true velocity, but also components arising from dynamics in the star's atmosphere, gravitational redshift, and other effects. For the Sun, such phenomena can be segregated since the relative Sun-Earth motion is known from planetary system dynamics. This is now becoming possible also for other stars, whose true radial motions are determined through space astrometry. A study of the differences between accurate astrometric velocities (from Hipparcos), and precise spectroscopic values (from ELODIE) is in progress. Data for cool stars in the Hyades indicate a tendency of relative blueshifts among earlier main-sequence F-type stars, and in giants. This is theoretically expected: an increased convective blueshift due to the more vigorous convection in F-stars, and a decreased gravitational redshift in giants.
\end{abstract}

\section{Introduction}

Non-spectroscopic determinations of radial velocities have hitherto been possible for solar-system objects, whose space motions can be derived with very high accuracy. For the Sun, this has permitted the segregation of wavelength-shift components originating from granular convection in the photosphere, gravitational redshift, pressure shifts, and other effects (Dravins 1999, and references therein). Astrometric techniques are now extending the realm of such geometrically determined radial velocities to many other nearby stars.

\section{Astrometric Radial Velocities}

The accuracies already achieved by Hipparcos (and especially those foreseen for future space astrometry missions) permit an accurate determination of stellar radial velocities without using spectroscopy or invoking the Doppler principle. However, among the several potential astrometric methods for deducing radial velocity (Lindegren et al. 1999; Madsen et al. 1999b), only that of changing angular extent of moving clusters yields sufficient accuracy already with current techniques to permit meaningful comparisons to precise spectroscopy.

${ }^{1}$ Based on data from the ESA Hipparcos astrometry satellite, and on observations collected at Observatoire de Haute-Provence. 
When a star cluster (whose stars share the same velocity vector) moves in the radial direction, its angular size changes, as measured by stellar propermotion vectors. This rate of change corresponds to the time derivative of the [known] distance, i.e., the radial velocity. Applying this method of changing angular extent, Hipparcos data have permitted accuracies of between 100 and $300 \mathrm{~m} \mathrm{~s}^{-1}$ to be reached for about one hundred stars in the nearby moving clusters of Hyades and Ursa Major (Dravins et al. 1997; Madsen et al. 1999a).

The possibility, in principle, of astrometrically determining radial velocities was realized already a long time ago (e.g. Schlesinger 1917), in particular the method of perspective acceleration, i.e. secular changes in the proper motion due to the changing perspective as the relative Sun-star distance varies due to stellar radial motion. That perspective acceleration was determined for Barnard's star by van de Kamp $(1967,1981)$ and for the white dwarf Van Maanen 2 by Gatewood \& Russell (1974). The latter case aimed at measuring the gravitational redshift through a comparison with spectroscopy. While the accuracies reached were perhaps adequate to detect the gravitational redshifts of white dwarfs $(\approx 30$ $\mathrm{kms}^{-1}$ ), they do not qualify as precise in our context (for Van Maanen 2 the radial velocity was obtained to a precision of $\pm 15 \mathrm{kms}^{-1}$ ), while the acceleration of Barnard's star calculated by van de Kamp (1981) corresponds to a standard error of $4 \mathrm{kms}^{-1}$.

\section{Gravitational Redshift}

The production of a redshift for photons escaping the potential well of solar gravity was predicted early in this century by Einstein, and numerous efforts have been made since to experimentally measure this quantity.

The gravitational redshift is given by the simple expression $V_{\mathrm{grav}}=G M / R c$, involving the constant of gravitation, the speed of light, and the stellar mass and radius. Using accepted solar values, we obtain $636.1 \mathrm{~ms}^{-1}$ for light escaping from the solar photosphere to infinity, and $633 \mathrm{~ms}^{-1}$ for light intercepted at the Earth. A spectral line formed at chromospheric heights ( $30 \mathrm{Mm}$, say), will have this shift decreased by some $20 \mathrm{~ms}^{-1}$, and a coronal line by perhaps $100 \mathrm{~ms}^{-1}$. For other stars, the shift scales as $\left(M / M_{\odot}\right)\left(R_{\odot} / R\right)$. This amount does not change very much for main-sequence stars between $\mathrm{A} 5 \mathrm{~V}$ and $\mathrm{KO} \mathrm{V}$ $\left(\approx 650 \mathrm{~ms}^{-1}\right)$, but reaches twice that value for the more massive early-B stars with $M \approx 10 M_{\odot}$. The extended dimensions of giants produce only much smaller shifts although, in their tenuous atmospheres, the exact amount of shift depends on the precise heights of line formation. Figure 1 attempts to summarize the expected gravitational redshifts in the Hertzsprung-Russell diagram, illustrating how the gravitational redshifts change by three orders of magnitude between white dwarfs and supergiants. This plot is not the result of modeling stellar masses and radii, but merely an illustration computed from standard stellar parameters.

Although the shifts are greatest in white dwarfs, their apparent faintness and paucity of sharp spectral features constrains any more detailed studies. However, such studies are feasible for components of common proper-motion systems, where the system velocity is spectroscopically determined (within $\approx$ $1 \mathrm{kms}^{-1}$ ) from the other component(s). Such studies (aiming for the determi- 


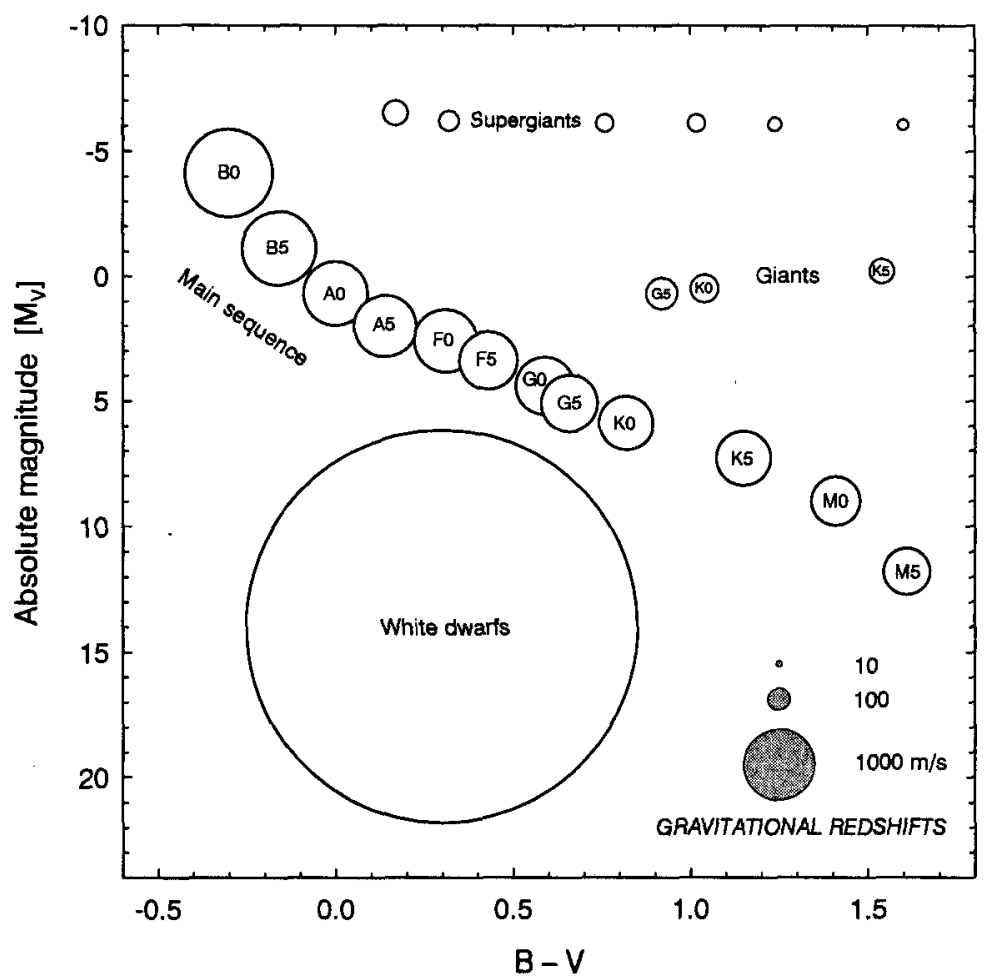

Figure 1. Theoretically expected gravitational redshifts in an HRdiagram, with the symbol area proportional to the amount of shift.

nation of white-dwarf masses) have been made by, for example, Bergeron et al. (1995) and Reid (1996).

If gravitational redshifts could be accurately determined for main-sequence stars, that would certainly improve the determination of their masses and radii (von Hippel 1996). However, contrary to what some authors have suggested, the main challenge is not to improve instrumental measuring precision, but rather to segregate the gravitational redshift from other physical phenomena affecting spectral line wavelengths.

\section{Spectroscopic Radial Velocities}

For cluster stars with astrometric radial-velocity determinations, a parallel spectroscopic program has recently been completed at Haute-Provence Observatory, using its ELODIE radial-velocity spectrometer connected to the 1.93-m telescope 


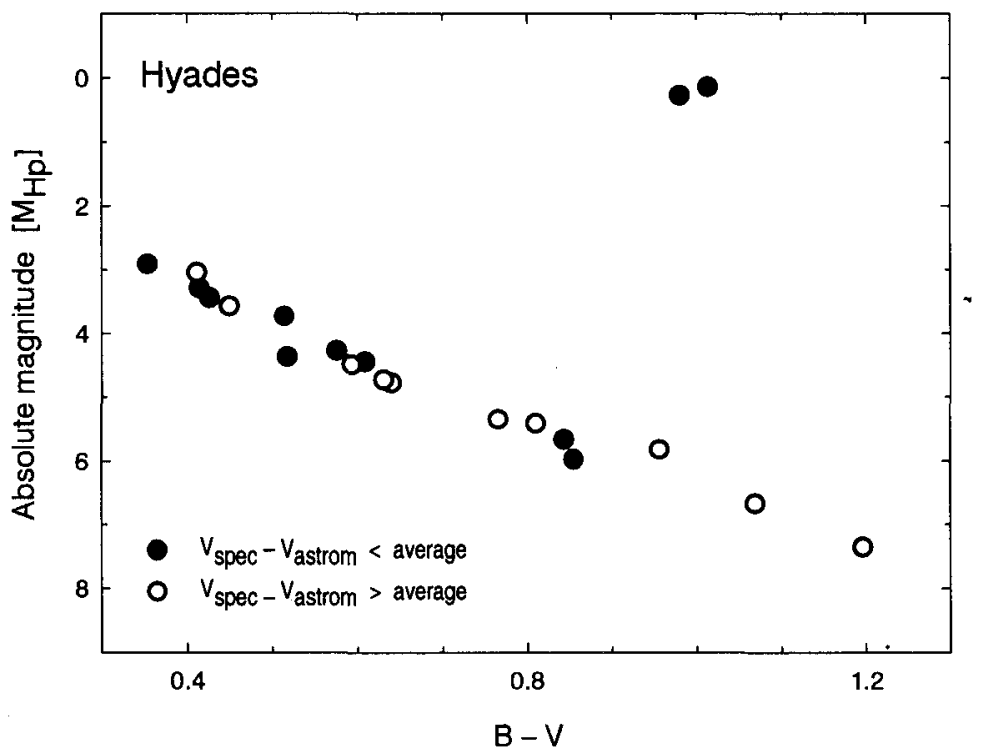

Figure 2. Differences between radial velocities in the Hyades, determined astrometrically (Hipparcos) and spectroscopically (ELODIE). Filled symbols denote stars whose spectra are blueshifted relative to the average, while open symbols show those with a relative redshift.

(Baranne et al. 1996). Its resolving power is about 40000 , and the spectral range $380-680 \mathrm{~nm}$ is covered in 67 échelle orders. Almost 100 program stars of many different spectral types were observed under generally very good signal-to-noise conditions. Besides the Hyades and Ursa Major stars in the astrometric program, the program also included several IAU radial-velocity standards, supplemented by observations of the Moon, metal-poor stars, Sirius, Procyon, and $51 \mathrm{Peg}$.

Work is in progress to compare the spectroscopic radial velocities with the astrometric values, and to search for systematic lineshift differences between groups of different spectral lines (with respect to line-strength, excitation potential, or wavelength region: Gullberg 1999a; 1999b). The overall stability of ELODIE spectra reaches $10 \mathrm{~ms}^{-1}$; the expected spectroscopic precision for groups of 100 selected lines in any one star is about $50 \mathrm{~ms}^{-1}$; the accuracy in astrometric radial velocity reaches $200 \mathrm{~ms}^{-1}$, while hydrodynamic models of stellar atmospheres predict differences on the order of $1 \mathrm{kms}^{-1}$ in convective shift between different stars (Dravins 1999). As already noted, gravitational redshifts are of comparable magnitude.

Spectroscopic radial velocities are obtained through software correlation between the recorded spectrum, and a reference spectrum template. The standard templates for ELODIE are based on masks for either spectral types F0 V or K0 III, produced from model atmospheres. In addition (for solar-type stars), we have used a mask based upon the solar flux atlas by Kurucz et al. (1984). The 
optimization of the extraction of spectroscopic velocities (including the design of correlation templates and calibration of their systematic shifts as function of, for example, rotational velocity) is in progress and will be reported elsewhere.

\section{Astrometry versus Spectroscopy}

The difference between $V_{\text {spec }}$ and $V_{\text {astrom }}$ contains components from: convective [blue?] shifts, atmospheric oscillations and pulsations, various activity-cycle effects, gravitational redshifts, and others. The gravitational redshift, $V_{\text {grav }}$, does not change much for main-sequence stars, but the convective shift, $V_{\text {conv }}$, is expected to be about $-1000 \mathrm{~ms}^{-1}$ for $\mathrm{F} 5 \mathrm{~V},-400 \mathrm{~ms}^{-1}$ for the Sun, and $-200 \mathrm{~ms}^{-1}$ for K0 V (Dravins 1999, and references therein). Other effects, such as pressure shifts appear to be small for ordinary stars (Allende Prieto et al. 1997), while isotopic shifts and effects of hyperfine structure can be avoided by a suitable choice of lines. The difference $V_{\text {spec }}-V_{\text {astrom }}$ can thus be expected to lie in the range between -400 and $+400 \mathrm{~ms}^{-1}$, moving along the main sequence from $\mathrm{F} 5 \mathrm{~V}$ to $\mathrm{K0} \mathrm{V}((B-V)$ between 0.4 and 0.9$)$.

Figure 2 shows the difference between astrometric and spectroscopic radial velocities, normalized to their average difference. A tendency is seen, such that relative blueshifts dominate among main-sequence stars of earlier spectral types $(\mathrm{F})$, and in giants. This is what is theoretically expected: the more vigorous convection in hotter stars causes an increased convective blueshift, while the increased physical size of giants decreases the gravitational redshift. The error budget, however, is rather complex, both for the astrometric and spectroscopic velocities, and will be discussed elsewhere. Since some part of the spectroscopic errors depends on spectral type (and on the stellar rotational broadening), the trends suggested by this figure should be viewed as preliminary only, until the analysis has been concluded.

\section{Conclusions and Future Work}

The astrometric radial velocity, at least in principle, appears to be a well-defined concept, although its determination may be coupled with practical challenges, such as the handling of binary or astrometrically otherwise complex stars.

In contrast, the spectroscopic radial velocity is not at all well-defined. It depends upon which lines are selected, precisely what parameter in which portions of each of these lines that is measured, and which wavelength region is examined. Furthermore, the line asymmetries and their wavelength measures depend on the stellar rotational line broadening, instrumental spectral resolution, and even the exact shape of the instrumental profile in the spectrometer.

On the theoretical side, gravitational redshifts (Figure 1) are, in principle, well-defined concepts. However, corresponding diagrams for the convective shifts cannot yet be produced, being physically very much more complex. Not only do these require a credible modeling of the hydrodynamics of stellar atmospheric fine structure, but the lineshift measures produced by such hydrodynamic atmospheres may vary greatly among different classes of spectral lines. 
However, all these dependences can be turned into an advantage. Since the exact values of spectroscopic radial velocities depend on so many parameters, stellar atmospheric properties can be constrained by different radial-velocity measures for the same star, or between different ones. Means toward this end include the optimal design of correlation templates, and the selection of spectral lines with sufficiently good laboratory wavelengths.

While the analysis is still very much in progress, the feasibility of accurate determination of astrometric radial velocities, and their meaningful confrontation with precise spectroscopic data has now been demonstrated. Looking to the more distant future, the accuracies will increase with future space astrometry missions, and with the availability of spectrometers combining very high spectral resolution with good wavelength stability.

Acknowledgments. This project is based on data from the ESA Hipparcos astrometry satellite, and on observations collected at Observatoire de HauteProvence. The work is supported by the Swedish Natural Science Research Council, the Swedish National Space Board, the Royal Physiographic Society in Lund, and the Anna-Greta and Holger Crafoord's Fund. Part of the material for this paper was prepared by D. Dravins during a sabbatical stay at the Institute of Astronomy, University of Latvia, Riga. A further discussion is available at URL: http://www.astro.lu.se/ dainis/

\section{Discussion}

Mazeh: You mentioned that youy measured the radiual velocity by fitting a parabola to the top of the peak. Did you try other methods?

Gullberg: The main reason for choosing this approach (fitting a parabola to the CCF) was to have a simple straight-forward method of error estimation that included the signal-to-noise ratio, etc. Results using gaussian fits and applying formulae in the literature did not give viable estimates.

\section{References}

Allende Prieto, C., García López, R. J. \& Trujillo Bueno, J., 1997, ApJ, 483, 941

Baranne, A., Queloz, D., Mayor, M., Adrianzyk, G., Knispel, G., Kohler, D., Lacroix, D., Meunier, J. P., Rimbaud, G. \& Vin, A., 1996, A\&AS, 119, 373

Bergeron, P., Liebert, J. \& Fulbright, M. S., 1995, ApJ, 444, 810

Dravins, D., 1999, IAU Coll 170, these proceedings

Dravins, D., Lindegren, L., Madsen, S. \& Holmberg, J., 1997, in Proc. Hipparcos - Venice '97, ESA SP-402, p.733

Gatewood, G. \& Russell, J., 1974, AJ, 79, 815

Gullberg, D., 1999a, IAU Coll 170, these proceedings

Gullberg, D., 1999b, IAU Coll 170, these proceedings 
Kurucz, R. L., Furenlid, I. \& Brault, J., 1984, Solar Flux Atlas from 296 to 1300 $\mathrm{nm}$, Sunspot, NM: National Solar Observatory

Lindegren, L., Dravins, D. \& Madsen, S., 1999, A\&A, in preparation

Madsen, S., Dravins, D. \& Lindegren, L., 1999a, A\&A, in preparation

Madsen, S., Lindegren, L. \& Dravins, D., 1999b, IAU Coll 170, these proceedings

Reid, I. N., 1996, AJ, 111, 2000

Schlesinger, F., 1917, AJ, 30, 137

van de Kamp, P., 1967, Principles of Astrometry, San Francisco: Freeman

van de Kamp, P., 1981, Stellar Paths, Dordrecht: Reidel

von Hippel, T., 1996, ApJ, 458, L37 